\title{
Video Article \\ Robotics and Dynamic Image Analysis for Studies of Gene Expression in Plant Tissues
}

\author{
Carlos M. Hernandez-Garcia ${ }^{1}$, Joseph M. Chiera ${ }^{1,2}$, John J. Finer ${ }^{1}$ \\ ${ }^{1}$ Department of Horticulture and Crop Science, The Ohio State University \\ ${ }^{2}$ Department of Plant Pathology, North Carolina State University
}

Correspondence to: John J. Finer at finer.1@osu.edu

URL: https://www.jove.com/video/1733

DOI: doi:10.3791/1733

Keywords: Plant Biology, Issue 39, gfp, image analysis, time-lapse animation, transient expression, promoters, transgene expression, transformation

Date Published: 5/5/2010

Citation: Hernandez-Garcia, C.M., Chiera, J.M., Finer, J.J. Robotics and Dynamic Image Analysis for Studies of Gene Expression in Plant Tissues. J. Vis. Exp. (39), e1733, doi:10.3791/1733 (2010).

\section{Abstract}

Gene expression in plant tissues is typically studied by destructive extraction of compounds from plant tissues for in vitro analyses. The methods presented here utilize the green fluorescent protein ( $g f p$ ) gene for continual monitoring of gene expression in the same pieces of tissues, over time. The gfp gene was placed under regulatory control of different promoters and introduced into lima bean cotyledonary tissues via particle bombardment. Cotyledons were then placed on a robotic image collection system, which consisted of a fluorescence dissecting microscope with a digital camera and a 2-dimensional robotics platform custom-designed to allow secure attachment of culture dishes. Images were collected from cotyledonary tissues every hour for 100 hours to generate expression profiles for each promoter. Each collected series of 100 images was first subjected to manual image alignment using ImageReady to make certain that GFP-expressing foci were consistently retained within selected fields of analysis. Specific regions of the series measuring $300 \times 400$ pixels, were then selected for further analysis to provide GFP Intensity measurements using ImageJ software. Batch images were separated into the red, green and blue channels and GFP-expressing areas were identified using the threshold feature of ImageJ. After subtracting the background fluorescence (subtraction of gray values of non-expressing pixels from every pixel) in the respective red and green channels, GFP intensity was calculated by multiplying the mean grayscale value per pixel by the total number of GFP-expressing pixels in each channel, and then adding those values for both the red and green channels. GFP Intensity values were collected for all 100 time points to yield expression profiles. Variations in GFP expression profiles resulted from differences in factors such as promoter strength, presence of a silencing suppressor, or nature of the promoter. In addition to quantification of GFP intensity, the image series were also used to generate time-lapse animations using ImageReady. Time-lapse animations revealed that the clear majority of cells displayed a relatively rapid increase in GFP expression, followed by a slow decline. Some cells occasionally displayed a sudden loss of fluorescence, which may be associated with rapid cell death. Apparent transport of GFP across the membrane and cell wall to adjacent cells was also observed. Time lapse animations provided additional information that could not otherwise be obtained using GFP Intensity profiles or single time point image collections.

\section{Video Link}

The video component of this article can be found at https://www.jove.com/video/1733/

\section{Protocol}

The following methodology outlines a protocol for image analysis of gene expression using an automated image collection system. For ease of explanation, the overall approach was broken down into four steps: 1) seed preparation, 2) gene introduction using particle bombardment, 3 ) robotic image collection, and 4) image analysis. Although this general methodology can be used for a broad-range of other applications, the present sets of protocols are based on the use of the green fluorescent protein (gfp) gene, which is a useful reporter gene for tracking gene expression in the same piece of living tissue over time.

\section{Seed Preparation}

1. Lima bean (Phaseolus lunatus cv. Henderson-Bush) seeds can be purchased, or ideally harvested from plants grown in a growth chamber ( $50 \%$ relative humidity, $16 / 8 \mathrm{~h}$ light:dark, $25 / 23^{\circ} \mathrm{C}$ day/night). Post-harvest seed quality may be maintained by storing seeds at $-20^{\circ} \mathrm{C}$.

2. Prepare Magenta GA7 containers for germinating seeds. Fold filter paper or paper towels to fit in the bottom of Magenta boxes. Add $\sim 25 \mathrm{ml}$ deionized water to each container to moisten paper towels, and pour off unabsorbed water. Autoclave moistened paper-containing boxes for $20 \mathrm{~min}$.

3. Using $50 \mathrm{ml}$ disposable centrifuge tubes, sterilize seeds in a $10 \%$ commercial bleach solution (20 seeds in $20-40 \mathrm{ml}$ ) for $20 \mathrm{~min}$ with shaking on a gyratory shaker at $60 \mathrm{rpm}$.

Note: All of the subsequent steps should be performed in a laminar flow hood.

4. Rinse seeds 4-7 times with sterile deionized water with gentle agitation for 30 sec during each rinse. 
5. Place 5-6 sterile seeds between layers of folded paper located in each sterile Magenta box.

6. Incubate seeds for 4 days under the following conditions: $25^{\circ} \mathrm{C}, 16 / 8 \mathrm{~h}$ light:dark and $40 \mu \mathrm{Em} \mathrm{s}^{-2}$.

\section{Gene Introduction using Particle Bombardment}

1. Build gene constructs using your favorite expression vector with a reporter gene that can be monitored in living tissues. We have engineered a high copy number expression vector useful for promoter and promoter element analyses. This vector contains the gfp gene which is used to visualize promoter/promoter element function in transformed tissues.

2. Around $1-2 \mathrm{~h}$ before bombardment, excise lima bean cotyledons from germinating seedlings and remove the seed coats. Cotyledons suitable for bombardment should be yellow to light green, flat and free of any damage that might interfere with further image analysis.

3. Place cotyledons on OMS culture medium containing MS salts ${ }^{1}, \mathrm{~B} 5$ vitamins ${ }^{2}, 3 \%$ sucrose and $0.2 \%$ Gelrite ( $\left.\mathrm{pH} 5.7\right)$ immediately after being excised.

4. Precipitate the DNA construct onto M10 tungsten particles (Sylvania, Towanda, PA, USA). In a $0.6 \mathrm{ml}$ microfuge tube, add $25 \mu \mathrm{l}$ tungsten particles (particles are resuspended in sterile water, $100 \mathrm{mg} \mathrm{ml}^{-1}$, right before use), $5 \mu \mathrm{l}$ DNA ( $\left.1 \mu \mathrm{g} \mu \mathrm{l}^{-1}\right), 25 \mu \mathrm{l} 2.5 \mathrm{M}$ calcium chloride (SigmaAldrich Cat. C3881-500G) and $10 \mu \mathrm{l} 100 \mathrm{mM}$ spermidine (Sigma Cat. S-2626). Vortex briefly to thoroughly mix all the components.

5. Incubate the DNA preparation on ice for $5 \mathrm{~min}$. Then, remove and discard $50 \mu \mathrm{l}$ supernatant.

6. Resuspend the DNA-coated particles by vortexing and immediately remove a $2 \mu \mathrm{l}$ aliquot. Repeat this vortexing step every time an aliquot is removed from the tube. Coated particles should be kept in ice and used within $15 \mathrm{~min}$.

7. Through the top of a syringe filter, place $2 \mu$ l coated particles in the middle of the filter screen. Place the filter unit containing the coated particles in the filter holding unit inside the particle gun chamber.

8. Place a lima bean cotyledon adaxial side up on a baffle and place the baffle in the particle gun chamber. The baffle, which consists of a screen melted to the bottom of a beaker, is used as a platform to support tissues during bombardment.

9. Bombard the tissues using a Particle Gun (in this methodology, we use a simple and inexpensive Particle Inflow Gun ${ }^{3}$, PIG, designed in our laboratory) as follows; a) open the valve leading to the vacuum to evacuate the chamber, b) after the vacuum reaches $760 \mathrm{~mm}(30 \mathrm{in}) \mathrm{Hg}$, activate the solenoid and release the helium to propel the particles, c) after particle bombardment, close the vacuum line valve and release the vacuum using the exhaust valve. The helium pressure used to accelerate particles is $50 \mathrm{PSI}$.

10. After the vacuum is released, open the chamber door to retrieve the bombarded cotyledons and return the cotyledon, adaxial side up, to OMS culture medium.

Note: For quantification and expression profiling, we usually bombard a minimum of three cotyledons for every DNA construct. A positive control, which is usually a DNA construct that gives a well-studied gene expression profile, is included as a reference.

\section{Automated Image Collection}

1. Turn on the mercury lamp and wait for $30 \mathrm{~min}$ for the lamp to warm up.

2. Turn on the power sources for the Spot-RT CCD camera (Diagnostic Instruments Inc., Sterling Heights, MI, USA) and the robotics platform motor controller, which drives the movement of the robotics platform.

3. Set the filter set for GFP detection (GFP2 filter set; Ex. $480 / 40$ nm, Em. 510 LP) on a MZFLIII dissecting microscope (Leica, Heerbrugg, Switzerland).

4. Sterilize the thickened polycarbonate Petri dish lids by spraying with $70 \%$ ethanol. The specialized lids, used to cover the Petri dish base prevent condensation during image collection ${ }^{4}$.

5. Place the plates containing bombarded cotyledons on the robotics platform (Arrick Robotics, Hurst, TX, USA) of the automated image collection system. The robotics platform is driven by a stepping motor set and consists of a table for attachment of 8 Petri dishes. The platform is constructed of polycarbonate, polypropylene and aluminum. Plates are fastened in position by tightening a lateral plastic setscrew.

6. Open the custom software application that controls both the robotics platform and image acquisition. Also, open the software white light controller switch.

7. In the "motor motion" tab, click on "motor on" and "motor home". After the software orients the platform to the home position, we are ready to start entering positions for every cotyledon.

8. In the "motor motion" tab, select the first plate. The platform will position the center of the Petri dish under the objective of the microscope. Using the moving distance buttons, precisely position the first cotyledon for image collection. With the white light controller on, the target region can be observed through the computer monitor by turning on the "live mode" feature.

9. For the first cotyledon, set the focus and magnification (1.6x magnification is preferred) using the manual knobs on the dissecting microscope. Focus adjustments for the remaining cotyledons on the same plate are made using three leveling screws located below each plate, on the robotics platform.

10. Once the region of interest has been set, click the "add this position" button. The software will add the coordinates of this region to a position schedule file and the platform will return to the center of the plate.

11. Set positions and focus for all the remaining cotyledons on plates using the moving distance buttons and manual leveling screws. After all the coordinates of all the cotyledons have been entered, save the position schedule coordinates.

12. Enter the image collection parameters. In the "image setting" tab, select the type of light desired; white or blue. For GFP detection, only the blue light should be used. Also, enter the exposure setting. Although we can modify time of exposure independently for red, blue and green color, we typically use the same settings for GFP photo-documentation (20,14 and $14 \mathrm{sec}$ for red, green and blue channels, respectively) allowing us to make direct and consistent comparisons among different DNA constructs.

13. In the same "image setting" tab, specify an empty folder where the images will be stored. Every series of images will be saved within this master folder in independent folders, numbered sequentially according to the order of positions entered for cotyledons.

14. Go to the "capture time control" tab and set the image acquisition time interval and total number of cycles of image collection. Images are typically collected every hour for $100 \mathrm{~h}$, generating well-defined gene expression profiles.

15. Start image acquisition by clicking on the "run schedule!" button located in the "position schedule" tab. 
16. At the end of the $100 \mathrm{~h}$ image collection, transfer all the images from the computer controlling the robot to any large capacity hard drive or computer for further image analysis. High-resolution images (1600 × 1200 pixels) are collected as TIF files measuring $\sim 5 \mathrm{Mb}$ each.

\section{Image Analysis}

1. Assemble the 100 sequential images from each folder using Adobe ImageReady.

1. Open ImageReady and import all the sequential images composing a series as frames.

2. Resize original images to $800 \times 600$ pixels. Collected images are high resolution, which generates large files which can make image processing slow.

3. Scroll through the images and find a spot(s) visible in most images.

4. Zoom up to $300 \%$ on the selected spot(s). Alignment of images using higher magnification allows for a more precise registration.

5. Insert a layer on top of all images and make sure is visible in all frames. Mark the spot(s) location using the "paintbrush" tool.

6. Start aligning all frames using the "move" tool and taking the marks on the layer as a reference.

7. When alignment is complete, delete the layer used as a reference and save images as a single "psd" file. Also, export all the frames as a single "mov" file using the highest resolution. Manual alignment of each image series takes $\sim 10$ min.

2. Perform quantification of gene expression using ImageJ.

1. Open ImageJ software and open the "mov" file saved previously in ImageReady.

2. Using the select tool, choose a $400 \times 300$ pixel area and crop the image. This new file should be saved as an "avi" file.

3. Separate the sequential images, located in the new "avi" file into red, blue and green channels by clicking "image", "color" and "RGB split" in ImageJ.

4. Subtract the background fluorescence from all images in the green and red channels. Using the green channel image series, select a $20 \times 20$ pixel area from a region with non-expressing cells and record its location in the "ROI Manager" tool, so that the same area will be selected in both channels. With the $20 \times 20$ pixel square active in the green channel, go to the "plugins" command located in the task bar of ImageJ and then click on the "subtract measured ROI for each slice" plugin-tool.

5. In ImageJ, click on "Image", "adjust" and then "threshold" to define or segment the GFP-expressing pixels. Adjust the threshold levels by dragging the bar in the threshold window to obtain an average spot size of 20-30 pixels.

6. Determine GFP expression using a plugin that we designed to measure the mean grayscale value per pixel and the total number of GFP-expressing pixels.

7. Copy the mean output grayscale values in ImageJ and paste into Microsoft Office Excel. Calculate the GFP expression for each channel (red and green) by multiplying the mean grayscale value per pixel by the total number of GFP-expressing pixels for each channel. The sum of expression values for both channels gives the GFP expression. Values can be used to illustrate gene expression profiles and make direct comparisons. Time-lapse animations can be also generated using "mov" or "avi" files, providing a unique and clear visualization of the gene expression profile.

\section{Representative Results}

The robotic image collection and analysis procedure (Fig. 1) reported here allows the acquisition of a large amount of quantitative data on gene expression in a short time period. For plant promoter characterization using the gfp gene, this methodology is not only useful to create transient expression profiles (Fig. 2) but also to track in a detailed-manner GFP expression in transiently- and stably-transformed plant tissues ${ }^{5,6}$. Short time-lapse animations generated with the collected sequential images are a valuable tool for in-depth analyses of gene expression over time in plant tissues. This methodology also has great application to evaluate factors that directly affect gene expression. For example, transient GFP expression under the presence of different suppressors of silencing of viral origin has been successfully studied using our automated image collection and analysis system ${ }^{7,8}$. 


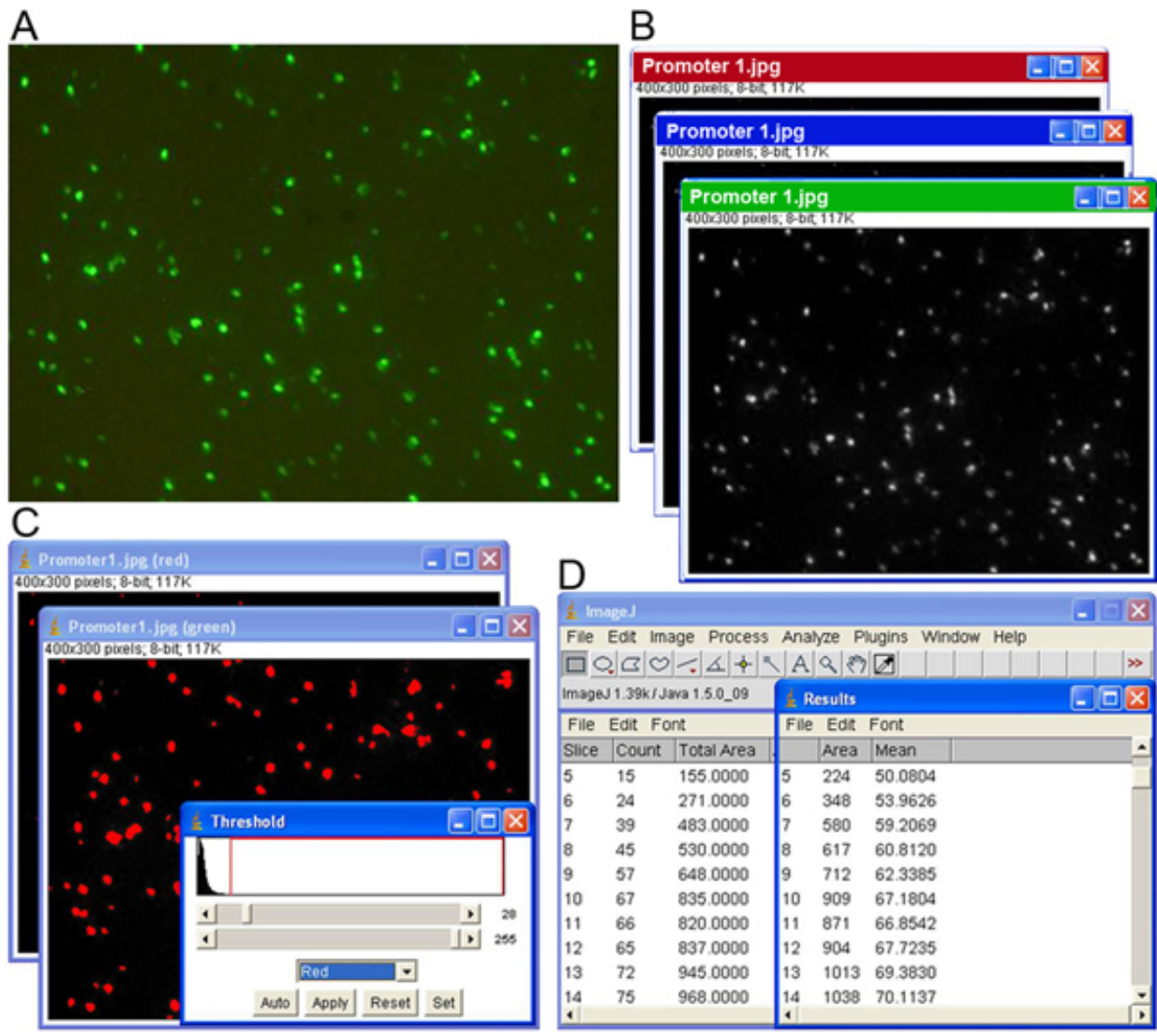

Figure 1. Image analysis procedure consists of four main steps. (A) Acquisition of image series using the robotic image collection system, (B) separation of images into red, blue and green channels, (C) segmentation of the expressing pixels by adjusting the threshold levels, and (D) obtaining the output results containing the grayscale values and the GFP-expressing focus counting.

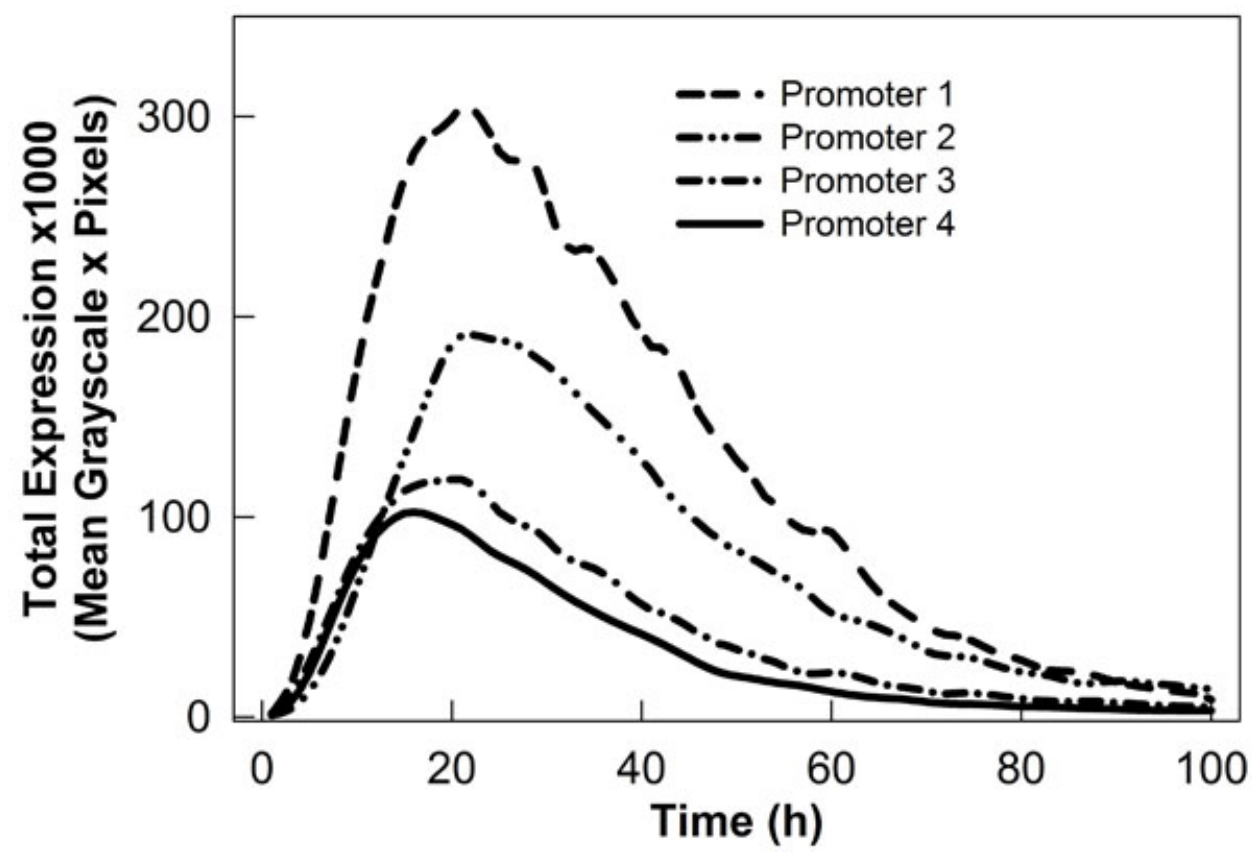

Figure 2. Graph showing different transient expression profiles driven by plant promoters fused to gfp. Data was collected using our robotic image collection system and analyzed with ImageReady and ImageJ software. 


\section{Discussion}

The use of robotics has enormous applications in different aspects of human life; specifically, robots have been effectively used to perform activities in dangerous environments, to automate tedious and complex activities, and to carry out tasks in a more precise way. In molecular biology, and specifically gene expression analyses, robots can help track not only features of genes but also tissue growth and development over time. Many biological phenomena occur dynamically, which may be difficult to follow using single time point observations.

The use of the gfp gene for expression studies brings additional advantages for observing tissue response and growth. For our experimental procedures, GFP allows us to follow gene expression in the same piece of tissue over time as GFP detection is a non-destructive. Furthermore, our version of the GFP protein is sufficiently stable to allow detection but also shows some turnover to minimize accumulation in plant tissues, allowing us to follow both the rise and fall of gene expression.

We have already utilized our robotic image collection and analysis system for a wide range of applications. We foresee high potential for many biological applications where a dynamic understanding of a phenomenon is desired. For example, growth and development of plant tissues can be tracked using our system giving valuable insight/information on these processes. Also, the dynamics of protein transport using reporter genes like $g f p$, can be easily visualized using time-lapse animations. The methodology described in this report is technically complex but conceptually simple. Our results are robust and new applications are continually being discovered.

\section{Acknowledgements}

Salaries and research support were provided by the United Soybean Board, and by State and Federal funds appropriated to The Ohio State University/Ohio Agricultural Research and Development Center. This research was also partially supported by a fellowship from CONACYT, Mexico, to CMHG. Mention of trademark or proprietary products does not constitute a guarantee or warranty of the product by OSU/OARDC and also does not imply approval to the exclusion of other products that may also be suitable. Journal Article No HCS 09-17.

\section{References}

1. Murashige, T. \& Skoog, F. A revised medium for rapid growth and bioassays with tobacco tissue cultures. Physiol Plant. 15, 473-497 (1962).

2. Gamborg, O.L., Miller, R.A. \& Ojima, K. Nutrient requirements of suspension cultures of soybean root cells. Exp Cell Res. 50, 150-158 (1968).

3. Finer, J.J., Vain, P., Jones, M.W. \& McMullen, M.D. Development of the particle inflow gun for DNA delivery to plant cells. Plant Cell Rep. 11, 232-238 (1992).

4. Finer, J.E. \& Finer, J.J. A simple method for reducing moisture condensation on Petri dish lids. Plant Cell Tiss Org. 91, 299-304 (2007).

5. Buenrostro-Nava, M.T., Ling, P.P. \& Finer, J.J. Comparative analysis of $35 \mathrm{~S}$ and Lectin promoters in transgenic soybean tissue using an automated image acquisition system and image analysis. Plant Cell Rep. 25, 290-296 (2006).

6. Chiera, J.M., Bouchard, R.A., Dorsey, S.L., Park, E.H., Buenrostro-Nava, M.T., Ling, P.P. \& Finer, J.J. Isolation of two highly active soybean (Glycine $\max (\mathrm{L}$.) Merr.) promoters and their characterization using a new automated image collection and analysis system. Plant Cell Rep. 26, 1501-1509 (2007).

7. Chiera, J.M., Lindbo, J.A. \& Finer, J.J. Quantification and extension of transient GFP expression by the co-introduction of a suppressor of silencing. Transgenic Res. 17, 1143-1154 (2008).

8. Dhillon, T., Chiera, J.M., Lindbo, J.A. \& Finer, J.J. Quantitative evaluation of six different viral suppressors of silencing using image analysis of transient GFP expression. Plant Cell Rep. 28, 639-647 (2009). 